\title{
Comparing the effectiveness of static stretching and proprioceptive neuromuscular facilitation stretching in treating delayed onset muscle soreness in calf muscles of runners
}

\author{
M. Ammar Ahmad Sohail, Ramesha Tahir $\bowtie$, Ayesha Maqbool $\bowtie$, Sana Hanif $\bowtie$, \\ Osama Saeed $\gg$ \\ Author affiliation: \\ School of Rehabilitation Sciences, The University of Faisalabad, Sargodha Road, Faisalabad, Pakistan. \\ Address: M. Ammar Ahmad Sohail; E-mail: ammar.sandhu666@gmail.com;_Telephone: 03041659795
}

\section{Abstract}

Objectives: To evaluate the comparative effectiveness of proprioceptive neuromuscular facilitation (PNF) and static stretching in relieving pain, increasing range of motion and improving functional disability in runners suffering from calf muscle delayed onset muscle soreness (DOMS).

Methodology: In this randomized controlled trial a sample size of 48 patients was taken from various gymnasiums of Faisalabad which were randomly allocated into 3 groups. Group $A(n=16)$ received static stretching, $G r o u p ~ B$ ( $n=16)$ received PNF stretching and Group $C(n=16)$ received no intervention being the passive control group. Patients' pain, range of motion (ROM) and lower extremity functional scale (LEFS) were assessed every $24 \mathrm{~h}$ after onset of DOMS for 5 days.

Results: No significant difference of age was observed between all groups. Significant improvement was demonstrated in all outcome measures across 5 days of treatment in subjects of all 3 groups with $p<0.05$ by using Repeated Measures analysis of variance (ANOVA). Results of one-way ANOVA demonstrated significant improvement in all outcome measures including pain, range of motion and lower extremity functional scale. But most significant improvement was observed in the PNF group.

Conclusion: PNF stretching has proved to be relatively more effective than static stretching in reducing pain and improving range of motion and lower extremity functional scale scores in runners suffering from calf muscle DOMS.

Abbreviations: PNF - proprioceptive neuromuscular facilitation; DOMS - delayed onset muscle soreness; ROM range of motion; LEFS - lower extremity functional scale; OTS - overtraining syndrome; EAMS - exercise associated muscle cramp; EIMD - exercise induced muscle damage; NPRS - numerical pain rating scale; HSD - "honestly significant difference"; GTO - Golgi Tendon Organs

Key words: Muscle soreness; Myalgia; Muscular cramp; Proprioceptive neuromuscular facilitation; PNF; Stretching; Muscle stretching exercise; Static active stretching

Citation: Sohail MAA, Tahir R, Maqbool A, Hanif S, Saeed O. Comparing the effectiveness of static stretching and proprioceptive neuromuscular facilitation stretching in treating delayed onset muscle soreness in calf muscles of runners. Anaesth. pain intensive care 2021;26(1):31-38. DOI: 10.35975/apic.v26i1.1763

Received: February 20, 2021, Reviewed: November 20, 2021, Accepted: November 22, 2021

\section{Introduction}

Delayed onset muscle soreness (DOMS) is characterized by pain, stiffness, tenderness, swelling and weakness of muscles, occurring as a result of arduous exercise performed by an individual. DOMS results in significant decrease in the muscle strength and range of motion (ROM) of the person. It takes $8 \mathrm{~h}$ for the torment to begin 
and a peak level of agony occurs 24 to $48 \mathrm{~h}$ post exercise, and it gradually returns to pre-exercise level. ${ }^{1}$

Various synonyms have been used by various authors to the phenomenon of DOMS, e.g. muscle fever, overtraining syndrome (OTS), post-exercise muscle soreness, exercise induced muscle damage (EIMD) and exercise associated muscle cramps (EAMC). ${ }^{2}$

Muscle damage after the unaccustomed strenuous physical activity is similar in males and females; however, the inflammatory response is exaggerated in women. Adults of all ages can develop DOMS. Pain from DOMS is stimulated by muscle palpation, stretch and muscle contraction and any mechanical stimuli that would usually not cause pain in a non-exercising individual. $^{3}$

The specific etiology of DOMS is unknown, yet but the following three theories have been suggested: metabolic waste accumulation theory which proposes that acute and delayed both kinds of muscle soreness occur as a result of lactic acid accumulation in the muscles after exercise. Muscle spasm theory suggests that ischemic pain is caused due to waste products build up in exercising muscles that leads to muscle spasm. The third theory presented by researchers suggests that contraction of the exercising muscles not only induces micro-tears in the muscle fibers but also causes damage to the connective tissue surrounding the muscle and the evidence of muscle damage is found by the increased level of plasma creatine kinase levels. ${ }^{4}$

DOMS significantly affects the functional status of an individual due to pain, hence the physical therapist is needed to combat the debilitating effects of DOMS. ${ }^{5} \mathrm{~A}$ few treatments options have been suggested e.g. pharmaceuticals, herbal, massage, hot/cold packs and nutritional supplements, but their supporting evidence is inconclusive. ${ }^{6}$ For a couple of years the use of unconventional cryotherapy has been proposed (extreme cold air exposure) or methods commonly used to relieve inflammation due to injuries for the treatment of DOMS; interval exercise and continuous exercise on DOMS and the conclusion was reached that there was no difference observed between the two treatments. ${ }^{8}$ So, it was demonstrated as a way to prevent or reduce the typical DOMS pain (or to accelerate the recovery), to stimulate muscles with lower volume eccentric exercises about a week or more, before the high volume eccentric training session. ${ }^{9}$

Proprioceptive neuromuscular facilitation (PNF) stretching is effectively being used to increase patients ROM to enhance motor function and rehabilitation as well as increasing the flexibility of the muscles. In this technique gait control theory, stress relaxation, reciprocal inhibition and autogenic inhibition are the main factors in increasing the ROM. ${ }^{10}$

DOMS has long been a source of concern for researchers, therapists and fitness professionals. It is one of the major dilemmas faced by patients performing demanding physical activity that fundamentally diminishes the exercise adherence. However, the effectiveness of stretching for DOMS is still debatable. The majority of studies on stretching and DOMS have looked at the effectiveness of static stretching. However, the effects of alternative stretch modes (for example, proprioceptive neuromuscular facilitation) on pain and functional deficits in DOMS are still unknown as only a few studies have looked at the efficacy of PNF stretching on DOMS. We examined the effects of PNF stretching and static stretching in DOMS in the calf muscles for treating pain, improving ROM and functional disability.

\section{Methodology}

A randomized control design study was conducted after getting approval from the ethical board of The University of Faisalabad. The University issued a data collection letter before conducting research and consent was also obtained from the managers of the gym settings. The study took 6 months duration to collect and analyze data after the approval of synopsis.

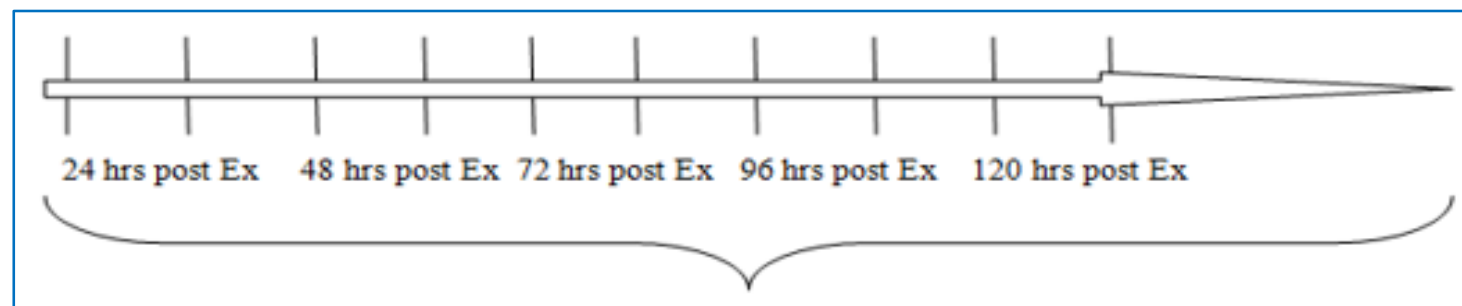

Figure 1: Experimental timeline

however, scientific studies have not been able to decisively demonstrate a significant difference in its treatment. $^{7}$

The researchers od an earlier study looked at the impact of low sets and repetitions performed at high intensity
Non probability purposive sampling technique was used to collect sample. Each patient gave informed consent for participation in research. Sample size was determined to be 48 with the assistance of the following formula by putting in values taken from previously published studies: ${ }^{11}$ 
$\mathrm{n}=2 \mathrm{SD}^{2}\left(\mathrm{Z}_{\alpha / 2} \pm \mathrm{Z}_{\beta}\right)^{2} / \mathrm{d}^{2}$

$* \mathrm{SD}=$ Standard Deviation, $\mathrm{d}=$ effect size $=$ difference between the mean values, $\mathrm{Z}_{\alpha / 2}=\mathrm{Z}_{0.05 / 2}=1.96$ (from $\mathrm{Z}$ table), $Z_{\beta}=Z_{0.20}=0.842$ (from $\mathrm{Z}$ table) at $80 \%$ power.

Patients were recruited from different gyms of Faisalabad, including Al Fahad Gym, Golds gym, Boulevard Gym and Zain Gym. Inclusion criteria comprised of age 18-45 y, DOMS in calf muscles, no known musculoskeletal disease, pain rating 3 to 8 on numerical pain rating scale (NRS) and Lower extremity functional scale (LEFS) score in range of 26 to 79. Patients taking anti-inflammatory medications or analgesics were excluded from the study, so there was no wash-out period. Patients who asked to take analgesics or other therapy during the intervention period were excluded to avoid the effect of any extraneous factor and solely obtain the results of the provided interventions. No other adjunct treatment was allowed despite of patient's pain and disability scores to avoid the effects of confounding factors.

The selected subjects were randomly allocated into three groups with 16 patients in each group. Randomization was performed through an online randomization generator. Group A was the static stretching group, Group B was the PNF stretching (holdrelax) group whereas the Group C was the passive control group.

To ensure double blinding the assessor was kept blind about the allocation of the patients. A second physical therapist assessed the outcome measures of VAS, ROM and LEFS. The patients were kept blind in this study about their assigned treatment group. Patients were definitely aware of what treatment they are receiving, but they weren't aware about which group they are in and what treatment is being provided in the other group. Thus, it was a patient blinded study.

Treatment groups followed the intervention plan; whereas passive control group was given no intervention to assess the phenomenon of natural recovery.

Group A performed static stretching of calf muscles; 10 repetitions with resting period of $10 \mathrm{sec}$ in between, twice per day for 5 days, holding each stretch for $30 \mathrm{sec}$.

Gastrocnemius Stretch: Patient placed non-stretched leg forward while keeping the heel of other leg on the floor. Patient shifted the body weight on the forward leg until the feeling of mild discomfort arose. Knee of the stretched leg was kept extended for gastrocnemius stretch.

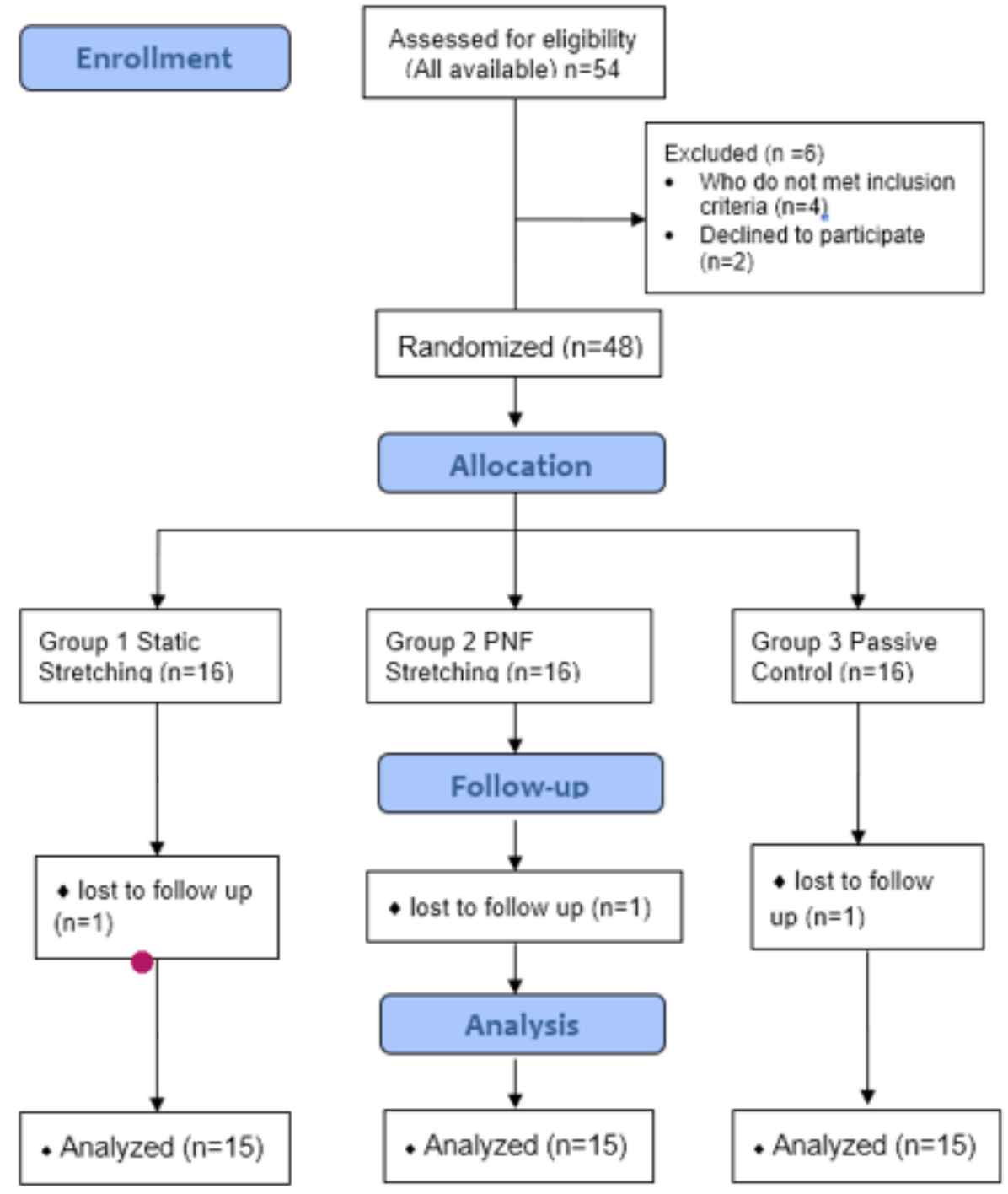

Figure 2: Consort Flow Diagram 


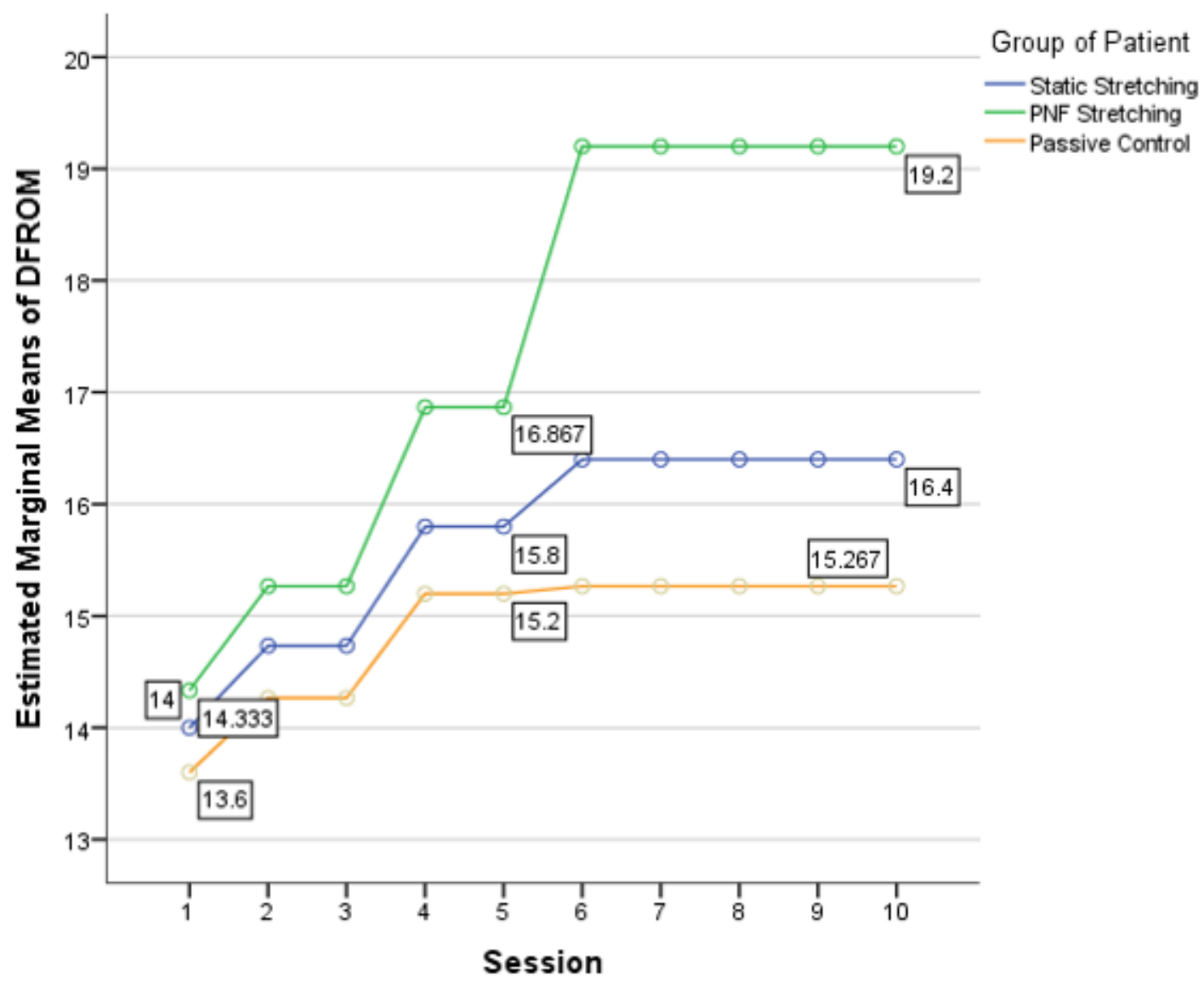

Figure 3: Graph showing mean improvement in dorsiflexion ROM between the groups

Soleus Stretch: Same procedure was repeated except that the patient position was with slight knee flexion instead of knee extension. ${ }^{1}$

Group B received PNF stretch of hold-relax-hold; the hold phase lasting $8 \mathrm{sec}$ and relaxation phase of $10 \mathrm{sec}$ performed 15 repetitions in one set for 5 days. In PNF stretching the range restricting calf muscle was elongated to the point of restriction at first and then a stretch was applied, followed by an end range isometric contraction for set time followed by voluntary relaxation to the tight calf muscles. At last, the limb was passively moved into the new range by the therapist. ${ }^{4}$

Group C (passive control group) was given no intervention. Protocol violation was dealt with intention to treat analysis. All participants were required to stop engaging in activities involving lower limbs.

VAS, goniometer and LEFS were selected as outcome measures depending on our feasibility, non-invasiveness, validity and reliability. Plasma creatine kinase was not chosen as an outcome measure as improvement in DOMS wasn't measured by changes in plasma creatine kinase levels, but was measured through different tools and scales which recorded subjective and objective changes. Universal Goniometer was used to measure ankle ROM in supine position. LEFS was used to measure lower limb function. Measurements of VAS and goniometry were taken pre- and post-treatment for 5 consecutive days. LEFS was assessed once a day for 5 days. Recruited participants were followed up for 5 consecutive days after every $24 \mathrm{~h}$. There was no observation or assessment period later on. Time points of VAS and ROM measurement with intervention given are shown below:

SPSS version 22 was used to manage and analyze the data. Data presentation was shown in the form of mean $\pm \mathrm{SD}$ along with $\mathrm{p}$-values. $\mathrm{P} \leq 0.05$ was considered as significant.

\section{Results}

Normality of data was analyzed before the application of statistical tests. Normality of data was checked through skewness, kurtosis and Shapiro-Wilk test values. For the sample size of less than 50, if the values of skewness and kurtosis are between +1.96 to -1.96 and $p$ value is more 
than 0.05 , then data is considered to be normally distributed. ${ }^{12}$

Values of data were falling between -1.96 to 1.96 and $p$ value $>0.05$ of Shapiro-Wilk's test showed that data for all outcome measures was normally distributed. As, data was found to be normally distributed, then parametric tests were applied. One way ANOVA was conducted to find between group difference among 1, 2 and 3 groups. Repeated measure ANOVA technique was applied to find within group difference for all outcome measures at different time points.

Mean ages of patients in group 1, 2 and 3 was $30.47 \pm 5.139$ y, $26.20 \pm 5.545$ y and $27.80 \pm$ 5.401 y respectively. P-value greater than 0.05 showed that there was no significant difference in mean ages in all groups.

Table 1 shows the inferential statistics for VAS, ROM and LEFS which were analyzed using repeated measures ANOVA. The table shows that there was significant improvement in all outcome measures across 5 days of treatment in subjects of all 3 groups with p value $<0.05$. Factor vs treatment group p-value is less than 0.05 which shows that there was difference in improvement of three groups.

Figure 3 illustrates mean improvement in dorsiflexion ROM measurement from baseline to
5 th day post treatment. The plot above shows increase in mean dorsiflexion ROM in all three groups. Significant improvement was observed in PNF stretching group considering the mean differences.

Results of one-way ANOVA demonstrated significant difference in dorsiflexion ROM measurement for three groups at day 3 post-treatment reading and day 4 and 5 with p-values less than .05. Post-hoc Comparisons using the Tukey HSD test indicated significant group differences for PNF group in dorsiflexion ROM in the follow up sessions with p-value less than 0.05 on day 2 , 3,4 and 5 .

\begin{tabular}{|c|c|c|}
\hline \multirow[t]{2}{*}{ Variable } & \multicolumn{2}{|l|}{ p-value } \\
\hline & Factor 1 & $\begin{array}{l}\text { Factor vs. treatment } \\
\text { group }\end{array}$ \\
\hline VAS & 0.000 & 0.000 \\
\hline Dorsiflexion ROM & 0.000 & 0.000 \\
\hline LEFS & 0.000 & 0.000 \\
\hline \multicolumn{3}{|c|}{$\begin{array}{l}\text { *VAS }=\text { Visual Analogue Scale, }{ }^{*} R O M=\text { Range of Motion, } \\
\text { *EFS }=\text { Lower Extremity Functional Scale, }{ }^{*} p \text {-value= } \\
\text { Probability value }\end{array}$} \\
\hline
\end{tabular}

\begin{tabular}{|c|c|c|c|c|c|}
\hline Variables & Time Points & $\begin{array}{l}\text { Static Stretching } \\
\text { Group }\end{array}$ & $\begin{array}{l}\text { PNF Stretching } \\
\text { Group }\end{array}$ & Control Group & p. Value \\
\hline \multirow[t]{10}{*}{ VAS } & Day 1 Pre Rx & $8.53 \pm .516$ & $7.93 \pm 2.251$ & $8.47 \pm .516$ & .427 \\
\hline & Day 1 Post Rx & $7.80 \pm .414$ & $7.00 \pm 2.000$ & $8.00 \pm .756$ & .082 \\
\hline & Day 2 Pre Rx & $7.53 \pm .516$ & $6.53 \pm 1.922$ & $7.67 \pm .617$ & .026 \\
\hline & Day 2 Post Rx & $6.80 \pm .676$ & $5.67 \pm 1.718$ & $7.40 \pm .632$ & .001 \\
\hline & Day 3 Pre Rx & $6.40 \pm .632$ & $5.00 \pm 1.558$ & $7.07 \pm .704$ & .000 \\
\hline & Day 3 Post Rx & $6.00 \pm .655$ & $4.53 \pm 1.407$ & $6.53 \pm .516$ & .000 \\
\hline & Day 4 Pre Rx & $5.53 \pm .640$ & $4.07 \pm 1.335$ & $6.33 \pm .724$ & .000 \\
\hline & Day 4 Post Rx & $5.00 \pm .756$ & $3.40 \pm 1.298$ & $5.87 \pm .640$ & .000 \\
\hline & Day 5 Pre Rx & $4.60 \pm .507$ & $2.67 \pm 1.047$ & $5.67 \pm .617$ & .000 \\
\hline & Day 5 Post Rx & $4.27 \pm .704$ & $2.07 \pm .799$ & $5.53 \pm .640$ & .000 \\
\hline \multirow[t]{5}{*}{ LEFS } & Day 1 & $48.27 \pm 14.315$ & $51.60 \pm 12.105$ & $49.67 \pm 13.798$ & .793 \\
\hline & Day 2 & $52.27 \pm 14.513$ & $57.53 \pm 12.744$ & $51.67 \pm 13.736$ & .441 \\
\hline & Day 3 & $54.93 \pm 14.305$ & $60.93 \pm 12.192$ & $53.53 \pm 13.010$ & .275 \\
\hline & Day 4 & $56.87 \pm 13.538$ & $63.67 \pm 10.861$ & $54.40 \pm 12.322$ & .114 \\
\hline & Day 5 & $58.60 \pm 12.293$ & $66.53 \pm 9.906$ & $54.87 \pm 11.087$ & .023 \\
\hline
\end{tabular}


Table 2 above shows the inferential statistics for the pain and LEFS, which were analyzed using one-way ANOVA. Results demonstrated significant difference in VAS scores at day 2, 3, 4 and 5 follow up session between the 3 groups with $\mathrm{p}<0.05$. Post hoc comparisons using the Tukey HSD test indicated significant group differences for VAS in PNF group in the follow up sessions with $\mathrm{p}<0.05$ on day 3,4 and 5 .

LEFS score demonstrated significant difference at day 5 with $\mathrm{p}<0.05$. The results were significant on day 5 as improvement in function took more time than improvement in pain. Post hoc comparisons using the Tukey HSD test indicated significant group differences for LEFS in PNF group in the follow up sessions with $\mathrm{p}$ $<0.05$.

\section{Discussion}

Current study was conducted to determine the comparative effectiveness of static stretching and PNF stretching for the treatment of calf DOMS in runners. The study evaluated the effect of these interventions on VAS, ROM and LEFS scores. Significant improvement of pain levels were found between 3 research groups after intervention from the baseline. The study demonstrated improvement in pain levels in all 3 groups. But significant improvement was recorded in the PNF stretching group.

An investigation on DOMS, treatment strategies and performance factors found consistent results as the present study. Results of the study demonstrated that stretching exercises were amongst the most effective option for reduction and symptoms of DOMS but this pain relieving effect was just temporary. ${ }^{13}$ Whereas, in contrast to present study, another RCT on the topic of the efficacy of Dynamic Contract-Relax Stretching on DOMS among healthy individuals concluded that pain did not decrease by performing post-exercise stretching. ${ }^{1}$

In the present study, significant improvement was demonstrated in dorsiflexion ROM from 3rd day post treatment till 5th day post-treatment. ROM scores increased in all groups. However, PNF stretching group showed significant improvement in dorsiflexion ROM.

Another research on the effect of PNF techniques on DOMS after Eccentric Exercise supports the findings of the current study by concluding that there was marked improvement in ROM levels after 48 and $72 \mathrm{~h}$ of PNF stretching. However, this study differs from the current study as the targeted area for treatment of DOMS was bicep brachii muscle instead of calf muscles. ${ }^{14}$

In contrast to our results, a study of the effect of PNF versus muscle energy technique (MET) in improving DOMS demonstrated different results, and concluded that there was improvement in ROM in both groups but more significant improvement was observed in MET group as opposed to significant improvement observed in PNF stretching group in our study. ${ }^{15}$

Another research on the effects of PNF stretching on post-exercise DOMS in young adults, and the correlation between the pre and post-exercise results upheld the results of the current study. They concluded that there was improvement in both ROM and pain levels for PNF stretching group in comparison to static stretching group. ${ }^{16}$ Whereas, another study demonstrated no improvement in flexibility (ROM) of muscles following 24 to $48 \mathrm{~h}$ after exercise, yet they reduced the muscle soreness. ${ }^{17}$

In the present study, LEFS scores showed significant difference in lower extremity function at 5th day of treatment between all groups. PNF stretching group showed significantly more improvement in LEFS scores compared to other 2 groups.

LEFS has not been assessed in any previous research which analyzed the effect of stretching exercises in treating DOMS. However, it had been used as an outcome measure in some studies which used other treatment strategies for DOMS. ${ }^{18,19}$

The benefits of static stretching (SS), ballistic stretching (BS), and PNF stretching on outcome measures like ROM have remained inconclusive in researches on stretches. PNF stretching has the potential to produce more improvements within participants' ROM, both active and passive. Autogenic inhibition, reciprocal inhibition, stress relaxation, and the gate control theory were recognized as the four possible mechanisms for ROM enhancement by PNF stretching. Each of these hypothetical mechanisms are reflexes that occur when the Golgi Tendon Organs (GTOs) in the tendons of the target muscle, or in the antagonist muscle to target muscle, perceive damaging stimuli (such as a stretching sensation). According to the findings, a combination of these four mechanisms improves range of motion. When this stretching technique is used after a workout, it improves functional performance and range of motion. ${ }^{20}$

\section{Limitations}

- $\quad$ Research was limited to calf muscle soreness only.

- Subjective measure like VAS and self-report questionnaire like LEFS are prone to be misinterpreted by the patients.

- Majority of study participants were young and trained athletes. So, results can't be generalized for elder and untrained persons with DOMS.

- Lack of interest from clients in performance of exercise due to their busy gym regimen. 
- Objective physiological measures like plasma creatine kinase to measure muscle damage were not taken due to limited economical resources.

- $\quad$ Limited number of new comers with new onset of DOMS and individuals with calf muscle soreness.

\section{Recommendations}

- $\quad$ New, large sample researches should be done using the same interventions on different muscles e.g. trapezius, deltoid etc. which may also be affected from DOMS after strenuous exercise.

- $\quad$ Research may be conducted on a mixed sample of both genders to see the effects of differences on DOMS and effects of interventions for DOMS.

- More objectives way can be used to assess DOMS like serum creatine kinase levels or infra-red technology.

- Future researches could add more outcome measures for detailed understanding of how DOMS affect human physiology.

- Different treatment options alone or in combination to these interventions can be used to treat DOMS in the future.

\section{Conclusion}

We conclude that PNF stretching and static stretching, both were found effective in reducing pain, improving range of motion and lower extremity functional scale (LEFS) score. But, most significant improvement was observed in PNF stretching group in decreasing pain scores, improving ankle range of motion and LEFS scores in calf muscle delayed onset muscle soreness in runners.

\section{Conflict of interest}

None declared by the authors

\section{Acknowledgement}

The authors express their sincere gratitude to the owners and the managers of the participating gymnasiums as well as the runners for allowing us to conduct this study.

\section{Authors' contribution}

MA, RT: Conception and design, Data collection and interpretation, Drafting of the article, Statistical analysis AM: Conception and design, Collection and assembly of data, Drafting of the article

SH: Conception and design, Critical revision of the article for important intellectual content, Final approval and guarantor of the article
OS: Critical revision of the article for important intellectual content, Final approval and guarantor of the article

\section{References}

1. Xie Y, Feng B, Chen K, Andersen LL, Page P, Wang Y. The efficacy of dynamic contract-relax stretching on delayed-onset muscle soreness among healthy individuals: A randomized clinical trial. Clin J Sport Med. 2018 Jan 1;28(1):28-36. [PubMed] DOI: 10.1097/JSM.0000000000000442

2. Qamar MM, Javed MS, Dogar MZ, Basharat A. Beat the exercise-induced muscle damage. JPMA. 2019 Nov;69(11):1682-85. [PubMed] DOI: 10.5455/JPMA.301636

3. Agten CA, Buck FM, Dyer L, Flück M, Pfirrmann CW, Rosskopf $A B$. Delayed-onset muscle soreness: temporal assessment with quantitative MRI and shear-wave ultrasound elastography. AJR. 2017 Feb;208(2):402-12. [PubMed] DOI: 10.2214/AJR.16.16617

4. Kisner C, Colby LA, Borstad J. Therapeutic exercise: foundations and techniques. Fa Davis; 2017 Oct 18. [Free full text]

5. Hohenauer E, Taeymans J, Baeyens JP, Clarys P, Clijsen R. The effect of post-exercise cryotherapy on recovery characteristics: a systematic review and meta-analysis. PLoS one. 2015 Sep 28;10(9):e0139028. [PubMed] DOI: 10.1371/journal.pone.0139028

6. Fleckenstein J, Niederer D, Auerbach K, Bernhörster M, Hübscher M, Vogt L, et al. No effect of acupuncture in the relief of delayed-onset muscle soreness: results of a randomized controlled trial. Clin J Sport Med. 2016 Nov 1;26(6):471-7. [PubMed] DOI: 10.1097/JSM.0000000000000259

7. Costello JT, Baker PR, Minett GM, Bieuzen F, Stewart IB, Bleakley C. Whole-body cryotherapy (extreme cold air exposure) for preventing and treating muscle soreness after exercise in adults. Cochrane Database Syst Rev. 2015 Sep 18; (9):CD010789. [PubMed] DOI: 10.1002/14651858.CD010789.pub2

8. Junior LF, Browne RA, Frazão DT, Dantas TC, Silva PH, Freitas RP, et al. Effect of low-volume high-intensity interval exercise and continuous exercise ondelayed-onset muscle soreness in untrained healthy males. J Strength Cond Res. 2019 Mar 1;33(3):774-82. [PubMed] DOI: 10.1519/JSC. 0000000000002059

9. Burt D, Lamb K, Nicholas C, Twist C. Lower-volume muscledamaging exercise protects against high-volume muscledamaging exercise and the detrimental effects on endurance performance. Eur J Appl Physiol. 2015 Jul 1;115(7):1523-32. [PubMed] DOI: 10.1007/s00421-015-3131-y

10. Wang $Y$, Xie $Y$, Feng B, Chen K. The efficacy of dynamic contract-relax stretching on calf muscle with delayed-onset muscle soreness in healthy individuals: a randomized clinical trial. Physiotherapy. 2015 May 1;101:e1603.

11. Charan J, Biswas T. How to calculate sample size for different study designs in medical research? Indian J Psychol Med. 2013 Apr;35(2):121-6. [PubMed] DOI: 10.4103/0253-7176.116232

12. Kim HY. Statistical notes for clinical researchers: assessing normal distribution (2) using skewness and kurtosis. Restor 
Dent Endod. 2013 Feb 1;38(1):52-4. [PubMed] DOI: 10.5395/rde.2013.38.1.52

13. Cheung K, Hume PA, Maxwell L. Delayed onset muscle soreness. Sports Med. 2003 Feb;33(2):145-64.

14. Lee SY, Yi CH, Choi MS. Effects of PNF technique on delayed onset muscle soreness after eccentric exercise. Phys Ther Korea. 2007;14(4):1-6. [Free full text]

15. Kalaiselvan A, MK M. Effect of proprioceptive neuromuscular facilitation versus muscle energy technique in improving muscle function in delayed onset muscle soreness in recreational players. Int J Pharma Bio Sci. 2017;8(3).

16. McGrath RP, Whitehead JR, Caine DJ. The effects of proprioceptive neuromuscular facilitation stretching on postexercise delayed onset muscle soreness in young adults. Int $\mathrm{J}$ Exerc Sci. 2014;7(1):14-21. [PubMed]
17. Ozmen T, Gunes GY, Dogan H, Ucar I, Willems M. The effect of kinesio taping versus stretching techniques on muscle soreness, and flexibility during recovery from nordic hamstring exercise. J Bodyw Mov Ther. 2017 Jan 1;21(1):41-7. [PubMed] DOI: 10.1016/j.jbmt.2016.04.001

18. Thiriot KN. The effect of photobiomodulation therapy on exercise-induced muscle damage. BYU; 2018. [Free full text]

19. Rizqi FY, Ambardini RL. The effectiveness of combination pnf stretching and cryotherapy in the prevention of DOMS in the lower extremities. Adv Soc Sci Edu Humanities Res. 2018;330(6):430-3. DOI: 10.2991/iceri-18.2019.90

20. Hindle KB, Whitcomb TJ, Briggs WO, Hong J. Proprioceptive neuromuscular facilitation (PNF): Its mechanisms and effects on range of motion and muscular function. J Hum Kinet. 2012 Mar;31:105-13. [PubMed] DOI: 10.2478/v10078-012-0011-y 